\title{
Design of the new type integrated-optical elements for E-field sensor
}

\author{
S.M. Kostritskii ${ }^{1}$, Yu.N. Korkishko ${ }^{1}$, V.A. Fedorov ${ }^{1}$, N.S. Korepanov ${ }^{1}$, \\ A.A. Dikevich ${ }^{1}$, P.Bourson ${ }^{2}$, M. Aillerie ${ }^{2}$ \\ ${ }^{1}$ MPTE Dept., Moscow Institute of Electronic Technology, Zelenograd, 124498, Russia \\ ${ }^{2}$ LMOPS, UMR CNRS 7132, Paul Verlaine University of Metz and Supelec, 2 rue E. Belin, \\ 57070 Metz, France \\ skostritskii@optolink.ru
}

\section{Introduction}

High voltage sensors that are based on electro-optic sensor heads have several advantages when compared with their purely electrical counterparts, for example minimal field disturbance, low and high frequency operation and immunity to electric noise and electromagnetic interference. Furthermore, when using an integrated scheme and optical fibre pigtailing they allow for both, a simple and compact design as well as excellent electromagnetic isolation.

Integrated optical sensors for the measurement of static and oscillating electric fields have been realized using different concepts. We propose to use the configuration based on an integrated Mach-Zehnder interferometer $(M Z I){ }^{1}$ in a material like lithium niobate, which provides large electro-optic effects as well as well-established methods for the fabrication of waveguide structures ${ }^{2}$.

It is a miniaturized sensor that does not require temperature compensation parts, provides a linear output, and the sensitivity of the sensor can be tailored over a large range. The sensor uses single-mode channel waveguide in $\mathrm{LiNbO}_{3}$ that are fabricated by proton exchange technique to guide light at wavelengths around $1.5 \mu \mathrm{m}$. Due to the linear electrooptic effect, the interferometer transmission is linearly changed as a function of electric field. Thus, for a fixed wavelength adjusted to an operating point in the curve of the transmission spectrum, the change in the relative phase shift provides a linear sensor output. To design advanced integrated-optic voltage sensor, we propose to fabricate the waveguide MZI in a proton-exchanged X-cut $\mathrm{LiNbO}_{3}$.

\section{Devices fabrication and characterization.}

The waveguide structure is a symmetrical Mach-Zehnder interferometer (MZI) with 6$\mu \mathrm{m}$-wide arms and two Y-junctions. The electrode structure consists of a coplanar waveguide with three electrodes. The width of the central electrode and the inter-electrode gap are chosen to be $18 \mu \mathrm{m}$ to arrive at a reasonable compromise between ohmic loss in the electrode and the impedance matching of the electrode structure to an external source. The total interaction length $\mathrm{L}$ is chosen to be $10 \mathrm{~mm}$. The necessary electrode pattern is photolithographically delineated in uniform Au-Cr seed layer that is deposited over substrate.

However, the lithium niobate waveguides have major drawback related to the photorefractive damage (PRD). Once PRD occurs, the MZI is biased at an incorrect point and the modulated signal includes severe non-linearity. Currently, the annealed proton exchange (APE) technique is used almost exclusively for the fabrication of optical waveguides in $\mathrm{LiNbO}_{3}$, owing to its simplicity. However, in addition to the PRD problem, treatment of a $\mathrm{LiNbO}_{3}$ substrate with benzoic acid produces severe crystal structure damage. The substrate must be annealed to reduce the damage. Problems caused by crystal damage include high propagation loss due to scattering, instabilities in the refractive index of the waveguide channels, and degradation of the electro-optic effect. Annealing the substrate minimizes these problems. One of the most important of such problems is DC stability. When an APE 
waveguide is utilized for the MZI, the MZI may need to be biased with an electric voltage to operate properly. For example, in the sensor application, a MZI needs to be biased at the socalled "quadrature" ( $\pi / 2)$ point to achieve maximum linearity. For a switch application, a bias voltage is needed to maintain the device in minimum or max imum light transmission.

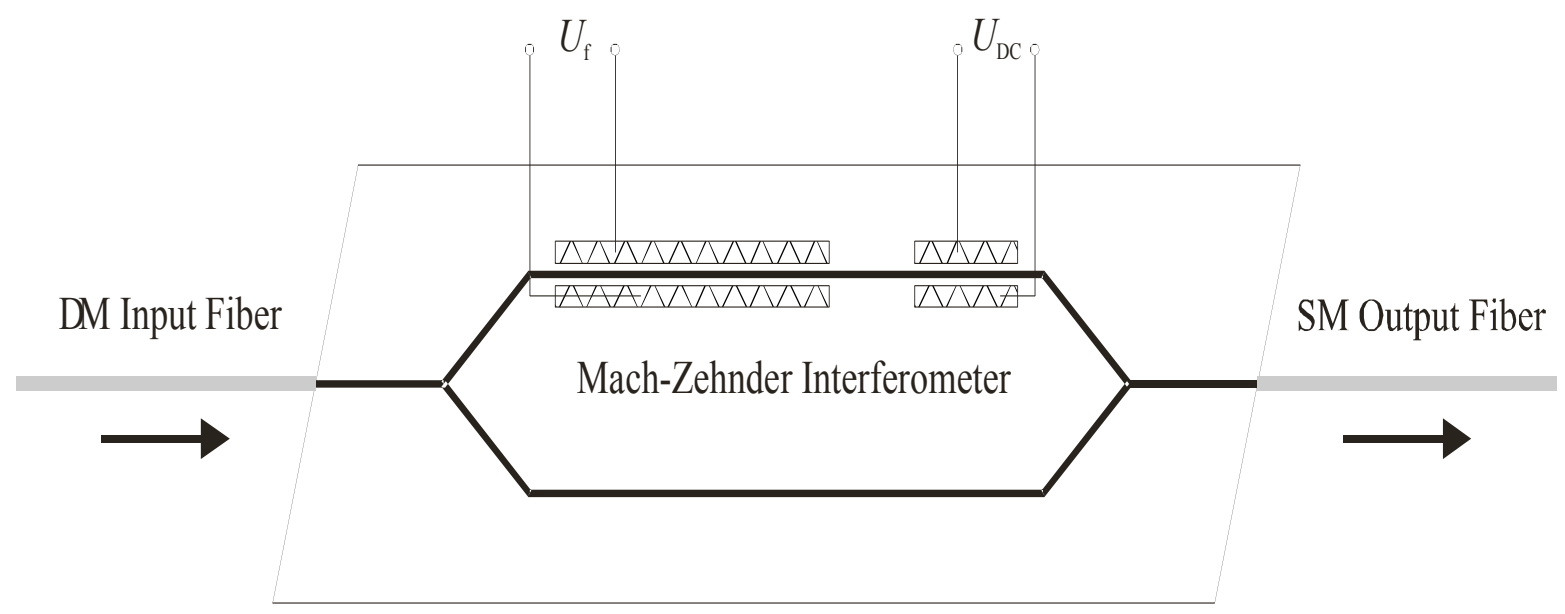

Fig. 1. Sensing integrated-optical element of E-field sensor.

After a $\mathrm{LiNbO}_{3}$ substrate is processed in a molten benzoic acid bath, the residual crystal damage and mobile charge migration present in the substrate cause the present bias point for the MZI to drift from the original set point of the $\pi$ phase over a period of time. This drift occurs at a very low frequency and is called DC drift. The period of time, which passes before such drift occurs, can be as short as a few minutes or as long as several hours. The DC drift may eventually even move beyond the maximum supplied voltage from the power supply.

DC drift is believed to occur because of the mobile charge and ions that are produced during treatment of a $\mathrm{LiNbO}_{3}$. When a bias voltage is applied to the modulator, the mobile charge and ions in the waveguide region slowly move toward opposing polarity electrodes and set up a counter electric field, which cancels the applied E-field from the electrode. The severity or magnitude of DC bias drift can vary among substrates processed in a molten benzoic acid bath. One solution to the DC drift problem is providing a mechanism for resetting the bias point with a refreshing voltage directed to the bias electrodes of the MZI. The refreshing voltage restores the MZI to its original setting. The utilization of a refreshing voltage, however, requires the use of a monitoring system to measure the drift and initiate the necessary resetting sequence. This makes the system design much more complicated and renders the $\mathrm{LiNbO}_{3}$ MZI useless for many applications. Accordingly, it would be highly desirable to provide an improved substrate and method for making the same, which would eliminate or minimize DC drift in the MZI.

To study electro-optical efficiency and DC-drift of the MZI fabricated at the different technological conditions, we have measured a switching voltage, i.e. half wave voltage $\mathrm{V}_{\pi}$. In our case of X-cut, Y-propagating geo metry of single-mode $\mathrm{PE} \mathrm{LiNbO}_{3}$ and Ti-indiffused (Ti: $\mathrm{LiNbO}_{3}$ ) waveguides the switching voltage of a MZI modulator is inversely proportional to effective value of electro-optic coefficient ${ }^{2} r_{33}$. Thus, if the overlap factor $F$ is known, $r_{33}$ can be determined from a single measurement of $\mathrm{V}_{\pi}$ :

$$
r_{33}=\left(\lambda G / n^{3}{ }_{e} V_{\pi} L F\right)
$$


where $\mathrm{L}$ is interaction length, i.e. length of electrodes in each interferometer arm, $\mathrm{G}$ - gap between electrodes, $\lambda$ - wavelength of guided light, what was $1534 \mathrm{~nm}$ in our experiments, $\mathrm{n}_{\mathrm{e}}$ -effective refractive index for $\mathrm{TE}_{0}$ mode.

The overlap factor $\mathrm{F}$ depends on the applied field and mode intensity profiles. The main uncertainty in $\mathrm{F}$ is due to random variation of mode profile, as the uncertainty of applied field profile can be eliminated entirely by choosing an electrode configuration, which produces an uniform electric field with in the waveguides. To realize this uniform field we have made Mach-Zehnder modulators with electrode gaps $\mathrm{G}=18 \mu \mathrm{m}$ much larger than the optical mode size $(\sim 4.5-6 \mu \mathrm{m})$. The electrodes are applied directly to the surface of the $\mathrm{LiNbO}_{3}$ substrate in order to eliminate errors caused by uncertainties in buffer layer thickness.

The both Ti:LiNbO 3 and APE $\mathrm{LiNbO}_{3}$ MZI modulators show marked drift in the DC bias voltage, $\mathrm{V}_{\mathrm{DC}}$, at the power levels on the order of $50 \mathrm{~mW}$ with no marked change of the modulation extinction ratio. Since the MZI modulator has an equal power in both arms, it is not as susceptible to photovoltaic induced refractive index changes as the directional coupler and phase modulator. At low and moderate input power levels (up to $100 \mathrm{~mW}$ ), the photoconductive damage gives dominating contribution in performance degradation of this device. The photoconductive damage manifests itself as a shielding of the applied DC field and a resultant shift in the DC bias voltage, $\Delta \mathrm{V}_{\mathrm{DC}}$, to a higher level to compensate. Indeed, this parasitic effect is larger and faster, when photoconductivity is increasing due to growth of either the light intensity I or the product of the absorption coefficient $\alpha$ with the photoconductivity coefficient $\beta_{\mathrm{ph}}$. Depending on $\mathrm{I}, \Delta \mathrm{V}_{\mathrm{DC}} / \mathrm{V}_{\mathrm{DC}}$ ranged from 0 to 0.2 and specific drift time was from 1 to 600 hours. The experimental samples of $\mathrm{APE} \mathrm{LiNbO}_{3} \mathrm{MZI}$ modulator exhibit the far superior photorefractive resistance and smaller DC-drift compared to $\mathrm{Ti}: \mathrm{LiNbO}_{3}$ modulators.

\section{Optimization of fabrication process with aid of Raman spectroscopy data.}

The severity or magnitude of PRD can vary among waveguides fabricated with different technological conditions. The choice of an optimal fabrication conditions, however, requires the rather simple characterization method to measure accurately the PRD. One from the most efficient techniques of such a characterization is the Raman spectroscopy, since the current studies have shown ${ }^{3}$ that PRD induces marked perturbations of a Raman signal in bulk crystals and $\mathrm{LiNbO}_{3}$ channel waveguides. Note also that, the optical requirements on a material for quantitative Raman data acquisition are often much less stringent than those for direct measurements of the PR effect. We demonstrate that the confocal micro-Raman spectroscopy method may be effectively used for accurate determination of PRD profiles in $\mathrm{LiNbO}_{3}$-based integrated-optical elements. We have recorded, see Fig.2, a Raman profile in direction perpendicular to the direction of the high-power laser beam, i.e. collinear to Z-ax is of X-cut crystal surface. The marked changes of Raman intensity were observed only near sharp boundaries between illuminated and non-illuminated areas, where the light-induced space charges are located and induce significant distortion of crystal structure ${ }^{3}$. Besides, the temporal evolution of Raman spectra between successive scans of the same area allows us to determine the PRD drift because of its specific kinetics ${ }^{3}$.

At the other hand, multiple confocal Raman spectra measurement at stepping translation of the microprobe beam within waveguide gives clear evidences of the specific PRD profile. The Raman scattering intensity as a function of the position of the scattering center within the optically damage path through the sample finally has been studied by this way. Raman intensity profiling $\mathrm{I}(\mathrm{Y}, \mathrm{Z})$ gives the important information necessary for comparative study of PRD in waveguides fabricated with different processing conditions. At the same time, our study opens the new possibilities for fast and easy estimation of 
performance of any newly developed LN-based materials (modified by doping or stoichiometry control) for application in integrated-optical sensing elements those feasibility is limited mainly by the PRD and light-induced contribution to the DC-drift effect.

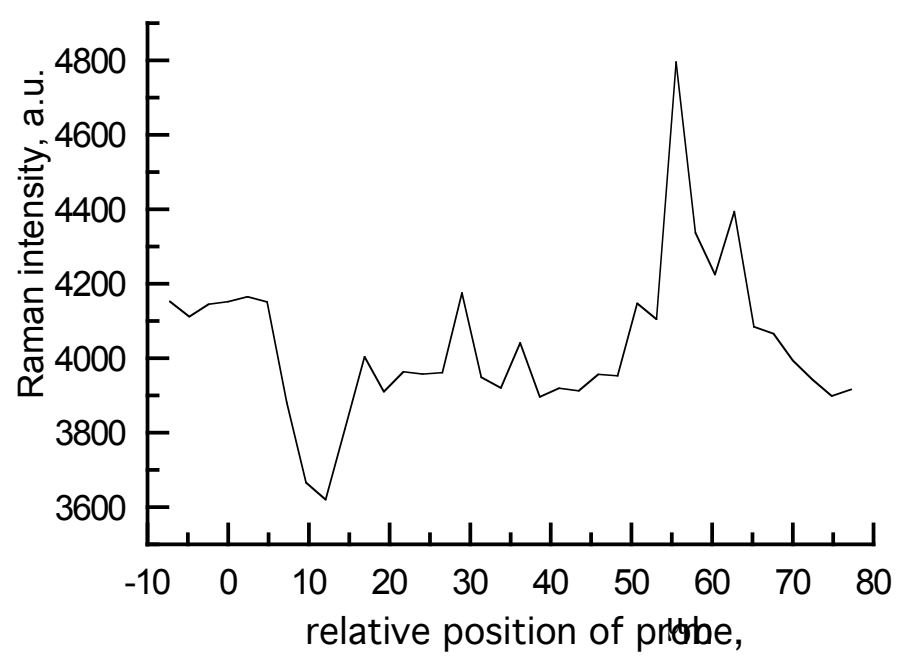

Fig. 2. PRD profile in a congruent $\mathrm{LiNbO}_{3}$ substrate, containing series of channel APE waveguides. Dependence of Raman intensity on the focal-point translation for a low-power $(10 \mathrm{~mW})$ probe beam. Raman spectra was obtained within the area damaged preliminary by the high-power $(320 \mathrm{~mW})$ illumination with focused $\mathrm{Ar}^{+}$-laser beam.

The phase composition of the novel waveguide structures formed with APE and soft proton exchange (SPE) ${ }^{4}$ has been determined by Raman spectroscopy data. The optimal fabrication conditions for obtaining homogeneous waveguiding layers via the PE techniques can be identified, using this method. It is important to note that even though $\alpha$-phase waveguides prepared by APE and SPE techniques have similar refractive indices and strains, there is likely to be a principal difference in the basic properties of the $\alpha$-phases as formed by these techniques. Indeed, it was reported recently that the dispersion of extraordinary refractive-index increment in $\alpha$-phase waveguides formed by SPE method was high compared to that in APE waveguides. Given these advantages of SPE in $\mathrm{LiNbO}_{3}$, the process is expected to improve the performance of a MZI sensing element as well. Since no attempts have been reported thus far, an investigation into this possibility is in order and presents an essential task of the proposed effort.

Finally, the fabrication conditions, used for soft proton exchange process and post exchange annealing, allowing to minimize PRD within waveguides and, thus, to decrease sufficiently the non-linearity of integrated-optical E-field sensor response (from 2.8 to $0.3 \%$ within $40-\mathrm{dB}$ voltage dynamic range) have been identified with aid of Raman spectroscopy data.

1. http://www.optolink.ru.

2. Yu.N. Korkishko, V.A. Fedorov, S.M. Kostritskii, A.N. Alkaev, E.I. Maslennikov, E.M. Paderin, D. Apraksin, and F. Laurell, Microelectron. Eng. 69, 228 (2003).

3. S.M. Kostritskii, P. Bourson, R. Mouras, and M.D. Fontana, Opt. Mater. 29, 732 (2007).

4. Yu.N. Korkishko, V.A. Fedorov, E.A. Baranov, M.V. Proyaeva, T.V. Morozova, F. Caccavale, F. Segato, C. Sada, S.M. Kostritskii, J.Opt.Soc.Am. A 18, 1186 (2001). 